\title{
LAS TEORÍAS NARRATIVAS FRENTE A LOS CASOS DE DUPLICACIÓN: UNA DEFENSA DE LA DETERMINACIÓN DE LA IDENTIDAD PERSONAL
}

\author{
NARRATIVE THEORIES AND DUPLICATION \\ CASES: A DEFENSE OF THE DETERMINACY OF \\ PERSONAL IDENTITY
}

\author{
Alfonso MuÑOZ CORCUERA* \\ Universidad Complutense de Madrid
}

Resumen: En este artículo argumento a favor de dos tesis. Primero, defiendo que las teorías narrativas de la identidad personal deben comprometerse con la intuición de que la identidad personal es determinada. Segundo, muestro cómo las teorías narrativas pueden ser exitosas en este empeño dando cabida a la dimensión social de nuestra existencia en tanto personas. Para organizar mi exposición me centro en uno de los casos más conflictivos para las teorías de la identidad personal: los casos de duplicación. Ante estos casos, las únicas opciones disponibles parecen ser bien aceptar que la identidad personal es indeterminada, o bien dar una respuesta arbitraria que permita conservar la determinación de la identidad a costa de vaciarla de toda su importancia práctica. Argumentaré por qué las teorías narrativas pueden escapar de ese dilema mediante una tercera vía.

\footnotetext{
* Profesor Ayudante Doctor. Departamento de Filosofía y Sociedad, Facultad de Filosofía, Universidad Complutense de Madrid, Ciudad Universitaria, Plaza Menéndez Pelayo s/n, 28040, Madrid, España. alfonso.m.corcuera@gmail.com.
} 
Palabras Clave: Identidad personal, teorías narrativas, intuiciones filosóficas, casos de duplicación, Parfit.

Aвstract: In this article I argue for two theses. First, I defend that narrative theories of personal identity must endorse the intuition that personal identity is determinate. Second, I show how narrative theories can succeed in this project by appealing to the social dimension of our existence as persons. To structure my discussion, I use one of the most troubling cases for personal identity theories: duplication thought experiments. Faced with those cases, the only available options seem to be either accepting that personal identity is indeterminate or give an arbitrary solution to the case that let us keep the intuition that personal identity is determinate. I will show why narrative theories can scape this dilemma thanks to a third alternative.

KeYwords: Personal identity, narrative theories, philosophical intuitions, duplication cases, Parfit.

\section{Introducción}

En este artículo argumento a favor de dos tesis:

(1) Las teorías narrativas de la identidad deberían defender de manera explícita que la identidad personal es determinada.

(2) Las teorías narrativas de la identidad pueden defender la determinación de la identidad aceptando lo que denomino la tesis social de la narratividad, que establece que la identidad personal se constituye a través de una negociación narrativa entre nuestra propia narración vital y las narraciones que los demás tienen sobre nosotros.

Con respecto a la primera tesis, me refiero a la cuestión de si la identidad personal es determinada (i.e., una cuestión de todo o nada, de tal manera que siempre debe haber una respuesta del tipo sí o no a las preguntas acerca de nuestra identidad) o indeterminada (i.e., una cuestión de grado, de tal manera que en ocasiones no es posible dar una respuesta definida a las preguntas sobre nuestra identidad). Hay que tener en cuenta que este tema ha recibido menos atención en las teorías narrativas que en otras aproximaciones al problema de la identidad personal. Pero esto no se debe a que haya ningún tipo de acuerdo al respecto. Existen narrativistas que defienden que la identidad es determinada 
(vid. MacIntyre, 2007), filósofos que toman por evidente que es indeterminada (vid. Dennett, 1991), y autores sobre los que es difícil saber qué opinan sobre el tema (vid. Bruner, 1986).

Con respecto a la segunda tesis es importante tener en cuenta que la tesis social de la narratividad puede ser interpretada como una tesis normativa, e incluso como una tesis metafísica, pero no debe entenderse como una tesis psicológica. Es una tesis acerca de en qué consiste nuestra identidad en tanto personas. Trata acerca de la cuestión metafísica de qué tipo de entidad son las personas y cómo se constituye su identidad en cuanto tales.

La estructura argumentativa de mi artículo será la siguiente: En la sección 2 explicaré por qué es importante para cualquier teoría de la identidad personal defender que ésta es determinada y señalaré por qué las teorías narrativas tienen un motivo adicional para comprometerse en la defensa de esta intuición. En la sección 3 presentaré brevemente las teorías narrativas de la identidad, introduciendo la tesis social de la narratividad. En la sección 4 mostraré cómo la tesis social de la narratividad puede ayudar a los narrativistas a defender que la identidad es determinada. En la sección 5, dado que la importancia de defender que la identidad personal es determinada depende parcialmente de la creencia de que la identidad personal es lo que importa cuando nos preocupamos por nuestra supervivencia, haré algunos comentarios en este sentido. Finalmente, en la sección 6 , considerando la importancia que otorgo a las intuiciones en mi argumentación, haré una reflexión sobre el papel de las intuiciones en la investigación filosófica a la luz de la filosofía experimental.

\section{La importancia de defender la determinación de la identidad personal}

Podemos empezar nuestra discusión a partir de un experimento mental que nos servirá para entender mejor el problema. En un futuro cercano, Bill es uno de tres gemelos idénticos. Un día los tres se reúnen para tomar café, con la mala suerte de que un coche los atropella, dejándoles gravemente heridos. Los médicos no creen que ninguno de los tres vaya a sobrevivir. Los cuerpos de los hermanos gemelos de Bill están en relativas buenas condiciones, pero sus cerebros están destruidos. Por su parte, el cuerpo de Bill no podrá recuperarse, pero su cerebro parece encontrarse en buen estado. En los últimos años ha habido grandes 
avances en el campo de la medicina, y los médicos piensan que podrían salvarle la vida a Bill trasplantando su cerebro al cuerpo de alguno de sus hermanos. El problema es que la operación tiene muy escasas probabilidades de éxito. Dichas probabilidades se multiplicarían por dos si en lugar de trasplantar el cerebro completo de Bill, separasen ambos hemisferios cerebrales y trasplantasen cada hemisferio al cuerpo de uno de sus hermanos. Los médicos le plantean el caso a Bill. La operación le costará todos sus ahorros. ¿Está dispuesto a someterse a ella? Bill duda: ¿y si los dos trasplantes son exitosos?

El experimento es una versión embellecida de un experimento mental que Parfit presentó en Reasons and Persons, el cual bautizó como "Mi división" (My Division) (Parfit, 1984: 254-255). Y apunta justo al problema que nos afecta: en determinadas situaciones, podemos saber todos los datos físicos y psicológicos relevantes sobre la situación de una persona y sin embargo no tener clara cuál sería la respuesta sobre su identidad. Si sólo un trasplante fuese exitoso, de acuerdo con Parfit, podríamos pensar que Bill sobreviviría a la operación, pues una persona puede sobrevivir con un único hemisferio cerebral. Pero si los dos trasplantes fuesen exitosos, ambos supervivientes serían dos personas distintas entre sí, por lo que Bill no puede ser idéntico a ambos. Pero si no es idéntico a ambos, ¿significa eso que no es idéntico a ninguno de ellos? ¿Implicaría un doble éxito en los trasplantes la muerte de Bill? Nuestra turbación surge, de acuerdo con Parfit, porque creemos que la identidad personal es determinada. Creemos que debe haber una respuesta clara, del tipo sí o no, a las preguntas sobre nuestra identidad. Sin embargo, concluye Parfit, no es así. La identidad personal es indeterminada, por lo que en ocasiones es imposible decir qué es lo que pasaría con Bill.

Volveré más adelante sobre lo que Parfit dice sobre este tema. Lo que ahora me gustaría destacar, sobre todo, es la insatisfacción que dicha respuesta le produciría a Bill. ¿Debería aceptar el doble trasplante? Desde luego, quiere sobrevivir, y está dispuesto a cualquier operación con tal de conseguirlo. Pero sólo si la operación efectivamente le permitirá seguir viviendo a él. No está dispuesto a dilapidar todo su dinero, a quitárselo a sus hijos, si el superviviente a la operación no fuese él, sino sólo alguien que se le parecerá mucho. Necesita tener certeza al respecto y una respuesta esquiva, que no sea del tipo sí o no, no será suficiente (vid. Williams, 1970: 174-175).

Esta necesidad de certeza surge de una de las características más básicas de la identidad personal: la preocupación egocéntrica (Schechtman, 1996: 2). Esto 
es, el tipo de preocupación específico que sólo podemos sentir hacia nosotros mismos. Bill sólo está dispuesto a someterse a la operación si efectivamente será él el superviviente. Si en el futuro, tras la operación, existirá una persona por la que tenga sentido que se preocupe egocéntricamente. Y sólo tendrá sentido preocuparse egocéntricamente por alguien si esa persona será idéntica a él. Nadie duda de la importancia de la preocupación egocéntrica para la identidad personal. Toda teoría de la identidad personal debería ser capaz de explicarla. Y si la preocupación egocéntrica nos hace pensar que la identidad personal debe ser determinada, toda teoría debería tratar también de dar cabida a esa intuición.

Volvamos un momento al experimento mental. Imaginemos que a Bill no le preocupa demasiado la posibilidad de duplicarse. Al fin y al cabo, las probabilidades dicen que es muy difícil que uno de los trasplantes salga bien. Que los dos trasplantes fuesen exitosos sería casi imposible. Pensando esto, Bill da luz verde a los médicos, con la mala suerte de que lo casi imposible sucede: tras la operación, los dos cuerpos de sus hermanos se despiertan, cada uno con la mitad del cerebro de Bill. Llamémosles BillC1 y BillC2. El caos se desata en ese momento. Ambos, BillC1 y BillC2, reclaman la identidad de Bill y, por tanto, los derechos y las responsabilidades asociados a la misma: su dinero, su trabajo, su familia... En este momento ya no se trata sólo de la preocupación egocéntrica de Bill. Todas las personas en la vida de Bill se ven afectadas por el problema. Su mujer no está dispuesta a estar casada con los dos supervivientes. En su trabajo necesitan saber a quién tienen que pedirle que vaya a la oficina el lunes. El hospital necesita saber a quién cobrarle la factura de la operación. En definitiva, la dimensión social de nuestra existencia nos reclama también defender que la identidad personal es determinada. En este sentido Cruz señala que "la sociedad ni tan siquiera puede funcionar sin el ineludible juego de adscripciones e imputaciones, reconocimientos y censuras, que vértebra las relaciones interpersonales" (Cruz, 2005: 48-49).

Lo dicho debería ser suficiente para afirmar que toda teoría de la identidad personal debería, como mínimo, intentar defender que la identidad personal es determinada. Pero como decía en la introducción, creo que las teorías narrativas están especialmente comprometidas en su defensa. El motivo se encuentra en sus propios orígenes, pues las teorías narrativas de la identidad surgen como una respuesta a las contraintuitivas conclusiones de Parfit (vid. e.g. Ricoeur, 1994: 129-139; Schechtman, 1996: 60-66).

De acuerdo con Parfit, las aproximaciones tradicionales al problema de la identidad personal intentan justificar dos de nuestras intuiciones más fuertes al 
respecto. La primera es que la identidad personal es lo que importa cuando nos preocupamos por nuestra supervivencia, de tal manera que la pregunta " $i$ sobreviviré?” es equivalente a la pregunta “¿existirá alguna persona que será idéntica a mí?”. La segunda intuición es la que nos ocupa en este artículo: la identidad personal es determinada, de tal modo que siempre debe haber una respuesta del tipo sí o no a la pregunta por nuestra supervivencia. Sin embargo, según Parfit, ambas intuiciones son erróneas (Parfit, 1984: 216-217), por lo que sería mejor abandonarlas.

Frente a esta postura, los primeros defensores de las teorías narrativas de la identidad pensaban que abandonar nuestras intuiciones era un precio demasiado caro por resolver el problema de la identidad personal. Especialmente en lo relativo a la intuición de que la identidad personal es lo que importa en la supervivencia. Pues, evidentemente, lo que nos importa cuando nos preocupamos por nuestra supervivencia es si nosotros mismos somos los que sobreviviremos. La existencia de una persona futura que se pareciese mucho a nosotros no sería suficiente para hablar de supervivencia. Al menos, no en el sentido de supervivencia que nos importa. De este modo, los narrativistas presentaron sus teorías como una alternativa a la propuesta de Parfit.

Como señalé en la introducción, la mayoría de los narrativistas no le prestan demasiada atención a la intuición de que la identidad personal es determinada. En sus críticas a Parfit se centran sobre todo en defender que la identidad personal es lo que importa. Sin embargo, si los narrativistas quieren que sus teorías sean una alternativa preferible a la postura de Parfit en cuanto a su contenido intuitivo, deben tratar de que su postura sea tan coherente con nuestras intuiciones como sea posible. Especialmente porque Parfit abre una puerta a conservar una de las dos intuiciones que rechaza. Precisamente la de la determinación de la identidad. De acuerdo con Parfit, dado que la identidad no importa, podemos enfrentarnos a los casos de indeterminación con una actitud pragmática y darles una solución arbitraria que nos permita tener una visión del mundo más coherente (Parfit, 1984: 240-241). En este mismo sentido Sanfélix señala que siempre podemos adoptar una convención que nos permita eludir la afirmación de que la identidad de una entidad es indeterminada (Sanfélix, 1994: 258). Por ejemplo, podemos volver al caso de Bill y decir que ninguno de los supervivientes sería idéntico a la persona original. O que el que tiene la identidad de Bill es BillC1 porque en el momento de la operación estaba más cerca de la ventana. Cualquier respuesta que diésemos sería igual de buena, pues en realidad no significaría nada. Sin embargo, nos permitiría conservar la intuición de que la 
identidad es determinada estipulando unas definiciones arbitrarias. En el caso de Parfit, su respuesta al caso de Bill sería decir que ninguno de los supervivientes a la operación es idéntico a Bill, pues para solucionar los casos de duplicación Parfit introduce una cláusula en su criterio de identidad que establece que las duplicaciones destruyen la identidad personal.

En este sentido, la teoría de Parfit es compatible con una de nuestras intuiciones más fuertes sobre el problema de la identidad personal. Por tanto, si las teorías narrativas quieren tener un mayor contenido intuitivo que la teoría de Parfit, deberían tratar de defender no sólo que la identidad personal es lo que importa cuando nos preocupamos por nuestra supervivencia, como ya hacen, sino también que es determinada.

\section{Las teorías narrativas de la identidad personal}

Antes de seguir adelante, necesito presentar otra pieza que será necesaria en mi argumentación: las teorías narrativas de la identidad personal. Se trata de una vertiente de las teorías de la identidad personal de inspiración lockeana que nació en los años ochenta del siglo veinte. Como Locke, los teóricos de la identidad narrativa defienden que hay una especial vinculación entre la identidad personal y una serie de problemas éticos y prácticos, como por ejemplo la cuestión de la supervivencia o la responsabilidad moral - aquí podemos mencionar la famosa aseveración de Locke de que "persona" es un término forense que imputa la responsabilidad de las acciones (Locke, 1975: 346)-. Al mismo tiempo, los narrativistas también están de acuerdo con Locke en que la mejor manera de capturar esa conexión entre la identidad personal y las mencionadas cuestiones éticas y prácticas asociadas a la misma consiste en entender la identidad personal en términos mentales. Locke hablaba de la continuidad de la conciencia (Locke, 1975: 344). Sin embargo, al contrario que otros teóricos neo-lockeanos como Parfit, los narrativistas consideran que no puede darse cuenta de esa continuidad de la conciencia a través de una explicación reductiva. Lo que constituye la identidad personal a lo largo del tiempo es la existencia continua de un mismo sujeto de experiencias que mantiene una conciencia unificada. En este sentido, los narrativistas consideran que nuestra vida mental tiene una dimensión específicamente subjetiva y no reducible que constituye la base de su unidad a través del tiempo. Los narrativistas caracterizan esta unidad subjetiva con lo que Strawson 
denomina la tesis psicológica de la narratividad, que establece que las personas experimentan su vida en forma narrativa (Strawson, 2008: 189).

$\mathrm{Al}$ respecto de la tesis psicológica de la narratividad es importante señalar que no se trata de una afirmación acerca de cómo las personas hablan sobre sus vidas. Es acerca de cómo las experimentan. En este sentido, la "narratividad" de la que hablan las teorías narrativas de la identidad personal no se refiere a la dimensión textual del término. De acuerdo con los narrativistas, experimentamos nuestra vida a través de narraciones en el sentido de que existe un principio organizador de nuestra experiencia, un filtro a través del cual pasan nuestras sensaciones para cobrar sentido (Schechtman, 1996: 113). Las afirmaciones de Bruner de que las narraciones son un modo de funcionamiento cognitivo (Bruner, 1986: 11), o las de Ricoeur acerca de cómo el tiempo se humaniza cuando se articula de un modo narrativo (Ricoeur, 1994: 52) deben entenderse desde esta perspectiva. Esto no significa que la narración a través de la que nos entendemos deba estar presente en la conciencia. O ser accesible en el sentido de que todos tengamos una historia de nuestra vida perfectamente articulada y lista para ser contada. Nuestra experiencia puede tener una estructura narrativa que opere de una manera inconsciente, y salir sólo a la luz en algunos momentos (vid. e.g. Jongepier, 2016). En este sentido, muchas de las críticas recibidas por las teorías narrativas en los últimos años tienen el problema de que no tienen una comprensión adecuada de en qué consiste la tesis psicológica de la narratividad (vid. e.g. Lamarque, 2007; Strawson, 2008).

Las teorías narrativas de la identidad personal se caracterizan igualmente por la defensa de una segunda tesis que no ha sido suficientemente señalada. Se trata de lo que denomino la tesis social de la narratividad, que establece que la identidad personal se constituye a través de una negociación narrativa entre nuestra propia narración vital y las narraciones que los demás tienen sobre nosotros. En ocasiones se caracteriza a las teorías narrativas somo si defendiesen que la identidad personal depende en exclusiva de la propia narración vital. Esta sería por ejemplo la caracterización que hace Strawson, y que resume en lo que denomina la tesis de la auto-constitución narrativa (Strawson, 2012: 74). Sin embargo, considero que esta caracterización es inadecuada. Por un lado, porque da pie a lo que Broncano denomina el problema del egocentrismo, confundiendo los conceptos de identidad personal y de autoconcepto (Broncano, 2013: 188). Y, por otro lado, porque la mayoría de los principales teóricos de la identidad narrativa asumen la tesis social de la narratividad. Seguramente es MacIntyre el autor que lo hace de una manera más explícita. Pero su postura al respecto es 
asumida como propia por otros narrativistas como Ricoeur (1994: 160), Taylor (2001: 36) o Rudd (2012: 178-179). Y sin citar a MacIntyre, también Dennett (1991: 429), Schechtman (2014: 103) y Lindemann (2014: 4) se han pronunciado en términos parecidos.

De acuerdo con MacIntyre, pasamos a formar parte de una narración desde el momento en que nacemos. Pero no es en nuestra propia narración en la que empezamos a existir, sino en las narraciones de otros. No aprendemos a hablar hasta muchos meses después de haber nacido, pero sería absurdo decir que no existimos en tanto personas antes de aprender a hablar. Nuestros padres nos dan un nombre y empiezan a contar historias sobre nosotros, a interpretar nuestro comportamiento y el modo en que afecta a sus vidas, desde el momento en que nacemos -o incluso antes-. Desde este punto de vista nos vemos forzados a reconocer que nuestra propia narración de vida se imbrica con otras narraciones de las que también formamos parte, constriñéndolas y siendo constreñida por ellas. Esto no quiere decir que debamos someternos al guion que los demás nos dan en sus narraciones. Seguimos teniendo libertad para actuar, del mismo modo que los demás personajes de nuestra narración escriben ellos mismos sus propias líneas en nuestra historia. En este sentido debemos admitir que nunca somos más que meros coautores de nuestra propia narración (MacIntyre, 2007: 213).

La postura de MacIntyre nos puede hacer pensar que la solución al problema del egocentrismo es irnos al otro extremo, y concebir la identidad como algo externo, que nos viene impuesto desde fuera. Incluso el propio MacIntyre parece inclinarse en este sentido, llegando a afirmar que las personas somos para siempre cualquier cosa que hayamos sido para los demás, pudiendo ser llamados a rendir cuentas de ello en cualquier momento (MacIntyre, 2007: 217). Esta postura podría permitirnos solucionar el problema que nos ocupa y defender que la identidad es determinada. La identidad personal vendría constituida por nuestro documento nacional de identidad, o cualquier otra adscripción que desde fuera nos pudieran imponer de una manera más o menos arbitraria. Sin embargo, considerar la identidad como el producto únicamente de las narraciones que tienen los otros no serviría para hacer justicia a nuestra existencia social, aunque hacerlo nos permitiese justificar que las personas puedan responder a la imputación de identidad determinada. En palabras de Cruz: 
No basta con disponer de un hipotético agente a quien endosarle la autoría de lo ocurrido: eso equivaldría a convertir el discurso ético en un ciego mecanismo de atribución de responsabilidades. Se precisa asimismo que haya alguien dispuesto a reclamar ese acto como suyo, a hacerse cargo de él. (Cruz, 2005: 52)

De este modo para definir a las personas nos vemos en la obligación de encontrar un equilibrio entre ambos polos. La negociación narrativa que da lugar a nuestra identidad según la tesis social de la narratividad tiene que partir del hecho de que ninguna de las dos posturas puede imponerse arbitrariamente a la otra. Por tanto, las creencias colectivas que dan lugar a dicha negociación tienen que contener la idea de que cada persona tiene una cierta autonomía, al mismo tiempo que también depende del resto de componentes del espacio social. "Autonomía que no puede eliminar la dependencia sin convertir al sujeto en un átomo autista; y dependencia que no puede ahogar la trayectoria del sujeto en un cúmulo de chantajes efectivos" (Broncano, 2013: 104). En una definición alternativa podemos decir entonces que ser persona "es moverse con adecuada habilidad entre dos fuentes de autoridad: la autoridad propia, el autogobierno o autonomía, y la autoridad del mundo, en el que cobran una especial relevancia los otros y la autoridad que implica su presencia” (Broncano, 2013: 69).

Muchos de los narrativistas que acabamos de citar parecen pensar que, en el fondo, nuestra propia narración y las narraciones que los demás tienen de nosotros son completamente coherentes entre sí. Sin embargo, como señala Goldie, nuestra mente es mucho más caótica de lo que los narrativistas suelen pensar (Goldie, 2012: 117-149). Y si prestamos atención a nuestra propia vida, no es difícil encontrar situaciones en las que nuestra propia narración difiere de las narraciones que los demás tienen sobre nosotros. Normalmente se trata de desacuerdos sin importancia -¿ocurrió el viernes o el sábado?-, de tal manera que una revisión superficial del caso nos permite alcanzar un acuerdo. Pero dado que la memoria no es un registro perfectamente fiable, siempre es posible encontrar versiones incompatibles de un mismo evento que difieren en aspectos esenciales. 
¿Qué sucede entonces con nuestra identidad? ¿Puede la tesis social de la narratividad ayudarnos a defender que la identidad personal es determinada?

\section{La tesis social de la narratividad y la determinación de la identidad}

Antes de mostrar cómo la tesis social de la narratividad puede ayudarnos a defender que la identidad personal es determinada, hay que hacer dos observaciones rápidas. La primera es que, pese a la multitud de ejemplos que pudiésemos encontrar en los que nuestra propia narración personal difiere de las narraciones que otros tienen sobre nosotros, todas las narraciones tienden a estar de acuerdo en los aspectos más importantes (dónde nacimos, cómo nos llamamos, quiénes son nuestros padres, a qué nos dedicamos...). Al fin y al cabo, son narraciones que tratan sobre los mismos hechos físicos en los que se vio involucrado un cierto ser humano. En este sentido, y si es necesario, siempre es posible recurrir a dichos hechos para alcanzar un acuerdo. La segunda observación es que el problema que nos ocupa es la cuestión de la identidad personal a través del tiempo. Evidentemente, es muy difícil alcanzar un acuerdo sobre todos los aspectos de la vida de una persona. Incluso puede que sea imposible alcanzar acuerdos sobre aspectos como su carácter. Pero esa no es la cuestión que nos ocupa. Lo único que pretendo defender es que, cuando nos preguntamos si una persona seguirá existiendo en el futuro o no, la respuesta a esa pregunta debe ser del tipo sí o no. Si hay otros aspectos de nuestra existencia que son indeterminados no es un aspecto que me concierna.

Aun teniendo en cuenta lo anterior, es posible pensar que una negociación narrativa podría concluir que la identidad de una persona es indeterminada. Sin embargo, aunque a priori podríamos pensar algo así, la tesis social de la narratividad implica que nuestra sociedad haría lo posible por alcanzar un acuerdo sobre la identidad de dicha persona, aunque fuese a posteriori. Imaginemos un juicio en el que el acusado se ha visto envuelto en una situación que nos hace dudar sobre si sigue siendo la misma persona que cometió el crimen o no. Es difícil imaginar que dicho juicio terminase con el juez afirmando que el acusado no es ni inocente ni culpable porque al mismo tiempo es y no es la misma persona que cometió el crimen. Dicha sentencia sería incoherente, o como mínimo altamente problemática. Por un lado, si el acusado queda en libertad tras el veredicto, no habría ninguna diferencia con decir que es inocente, esto es, que no es la misma 
persona que cometió el crimen. Pero por el otro lado, si el acusado es enviado a prisión, sería muy difícil justificar que está siendo castigado pese a no ser la persona que cometió el delito. Todo nuestro sistema de derecho se basa en la idea de que las personas sólo son responsables de sus propios actos. Es radicalmente injusto castigar a alguien por un crimen cometido por otra persona. Siendo así, si el acusado es enviado a prisión, el juez estaría obligado a justificarlo diciendo que el acusado es la misma persona que cometió el crimen. Alguien podría argumentar que el juez podría enviarle a prisión, pero con algún atenuante, admitiendo así que en parte el acusado no es la misma persona que cometió el crimen. Sin embargo, una sentencia de ese tipo seguiría sin ser justa. Si el acusado no es de una manera clara e inequívoca la misma persona que cometió el crimen, castigarle, aunque sea un castigo leve, sería injusto, del mismo modo que sería injusto castigar al hermano de un asesino, aunque fuese sólo a un día de prisión.

Sabiendo que la tesis social de la narratividad implica que la negociación narrativa que constituye la identidad de una persona no puede aceptar que su identidad sea indeterminada, es momento de volver al caso de BillC1 y BillC2. ¿Alguno de ellos es idéntico a Bill? Obviamente no puedo dar una respuesta definitiva al problema, pues no puedo saber qué dirían las distintas voces que intervendrían en la negociación narrativa que establecería la identidad de Bill y sus descendientes. Sin embargo, sí puedo explicar cómo podría desarrollarse dicha negociación para llegar a una conclusión.

Lo primero que debemos señalar es que el caso no podría resolverse sin tener en cuenta a BillC1 y BillC2, por lo que será necesario atender a sus condiciones. Cuando Parfit presenta el experimento "Mi división", parte del supuesto de que es posible que los hemisferios cerebrales de una persona sean funcionalmente idénticos y que por tanto los dos descendientes del experimento sean completamente continuos con la persona original desde el punto de visto psicológico (Parfit, 1984: 255). Sin embargo, se trata de un supuesto altamente cuestionable. Por un lado, porque los hemisferios cerebrales suelen tener funciones distintas. Y segundo, porque la posesión de un único hemisferio cerebral disminuiría significativamente las capacidades mentales de los dos descendientes.

Este aspecto podría utilizarse para negar la utilidad del experimento mental de Parfit. Se trataría de un experimento mental defectuoso que no produce resultados fiables para la investigación en identidad personal (vid. Wilkes, 1988). Sin embargo, el problema es fácilmente subsanable, pues Parfit no necesita que su experimento implique que los dos hemisferios cerebrales de una persona sean 
funcionalmente idénticos. Lo único que necesita defender es que, tras el doble trasplante, cada hemisferio cerebral daría lugar a un sujeto de experiencias con las capacidades mentales necesarias como para alcanzar la condición de persona y con una continuidad psicológica con Bill lo suficientemente elevada como para que sea pertinente preguntarnos si es idéntico a Bill o no. Este supuesto es plausible, pues parece coherente con nuestras creencias y prácticas actuales relativas a aquellos sujetos a los que, por un motivo médico, se les extrae un hemisferio cerebral. En dichos casos, todo parece indicar que la posesión de un único hemisferio cerebral no es impedimento para que consideremos que, tras la operación, los pacientes siguen siendo sujetos de experiencias, siguen siendo personas y siguen conservando la misma identidad que tenían antes de la hemisferectomía. Siendo así, no parece descabellado presuponer, en el experimento mental que nos ocupa, que cada uno de los hemisferios cerebrales de Bill podría dar lugar a un sujeto de experiencias con las capacidades mentales necesarias para alcanzar la condición de persona y con una continuidad psicológica con Bill lo suficientemente elevada como para que sea pertinente preguntarnos si es idéntico a Bill o no. Lo único que debemos considerar es que, dado que los dos hemisferios cerebrales no son idénticos entre sí, BillC1 y BillC2 tendrán propiedades mentales distintas, pudiendo diferir en sus opiniones acerca de cualquier cosa.

Considerando lo anterior, podemos ahora volver atrás y entender por qué decía que lo primero que debemos tomar en cuenta para analizar el problema de la identidad de Bill será lo que BillC1 y BillC2 tengan que decir sobre su propia identidad. Quizá ambos reclamen la identidad de Bill, como asume Parfit. Pero es concebible que no lo hagan. Quizá experimentar una situación tan radical les haga plantearse que su existencia actual no tiene nada que ver con la vida anterior de Bill. Incluso podría ser que, a pesar de ser ambos psicológicamente continuos con Bill, BillC1 y BillC2 experimenten de manera distinta su pasado, de tal manera que sólo uno de ellos se sienta efectivamente idéntico a Bill. Este será un aspecto que la negociación narrativa que establezca la identidad de ambos deberá tener en máxima consideración.

No obstante, lo que BillC1 y BillC2 digan no será la última palabra sobre el caso. Como decía anteriormente, la tesis social de la narratividad implica que la identidad se constituye en la negociación entre la propia narración y las narraciones que los demás tienen sobre el sujeto. En este sentido será necesario que el punto de vista de primera persona de BillC1 y BillC2 entre en diálogo con lo que el resto de la sociedad tenga que decir al respecto. 
Imaginemos que un grupo de científicos anuncia que han desarrollado la técnica que permitiría salvar la vida a Bill: son capaces de separar los dos hemisferios cerebrales de una persona y trasplantarlos por separado a cuerpos distintos. Tras el anuncio, sin duda, las discusiones sobre el caso se multiplicarían. No sólo en el ámbito filosófico, como sucede en la actualidad, o entre médicos, como sería esperable. Un avance técnico de este calibre, con las implicaciones que tendría, llegaría a los medios, y a través de ellos a la calle, donde la gente comenzaría a pensar sobre el caso y a discutir sus implicaciones. Sin duda se crearían comités éticos para estudiar si una operación así debería permitirse, y bajo qué condiciones. Y en los ámbitos político y jurídico se pediría ayuda a médicos y filósofos para dilucidar qué sucedería con los sujetos que sufriesen una operación de ese tipo. Estos debates sin duda serían variados y previsiblemente con pocos puntos en común, pues sin la existencia de un caso actual de una persona que se sometiese a la operación no existiría presión alguna para llegar a acuerdos.

Sería entonces la existencia de un caso actual, la existencia de BillC1 y BillC2, la que llevaría ese debate al siguiente nivel, dando lugar a una genuina negociación narrativa sobre sus identidades. La existencia de los dos sujetos supondría una presión suficiente como para forzar la necesidad de un acuerdo. Dicho acuerdo sin duda partiría de lo que BillC1 y BillC2 tuviesen que decir sobre sí mismos. Pero también tendría en consideración otros aspectos. Por ejemplo, el estado de salud de ambos sujetos, la opinión de la familia de Bill o las repercusiones éticas, legales y políticas que pudiesen tener dichos juicios de identidad. Es difícil saber cómo se desarrollaría el debate para llegar a un acuerdo al respecto, pero mi intuición es que finalmente dicho acuerdo se tendría que realizar a un nivel legal. Seguramente habría un juicio para tratar de esclarecer los muchos aspectos de la vida de Bill que habrían quedado en el caos, y finalmente una ley regularía las operaciones de doble trasplante de los hemisferios cerebrales de una persona para evitar que dicho caos pudiese producirse de nuevo en el futuro. De este modo, la ley eliminaría la indeterminación de la identidad que se produciría en dichos casos, refinando nuestra definición del concepto de persona.

\section{Lo que nos importa en nuestra supervivencia}

Hasta ahora he mostrado cómo las teorías narrativas pueden defender que la identidad personal es determinada gracias a la tesis social de la narratividad. Sin embargo, mi estrategia argumentativa se parece mucho a la estrategia utilizada 
por Parfit para mantener esta misma intuición. Como señalé más arriba, de acuerdo con Parfit sería posible preservar la determinación de la identidad personal estableciendo una convención arbitraria que nos permita resolver los casos conflictivos. Pero hacer depender la identidad de una decisión arbitraria parecería implicar un vaciamiento de la importancia que normalmente atribuimos a la identidad personal. Especialmente en lo tocante a lo que nos importa cuando nos preocupamos por nuestra supervivencia. Este aspecto no representa un problema para Parfit, pues él ya sostiene por otras razones que la identidad personal no es lo que importa cuando nos preocupamos por nuestra supervivencia. Sin embargo, sí sería un problema para los narrativistas, pues su compromiso es otorgar un mayor contenido intuitivo a su propuesta que el que tiene la teoría de Parfit. En este sentido, resulta necesario decir algunas cosas a este respecto para mostrar cómo es posible para los narrativistas defender la determinación de la identidad sin renunciar a la intuición de que la identidad personal es lo que importa.

En primer lugar, es importante señalar que hacer depender la identidad de una negociación narrativa -o, en última instancia, de una ley- no significa hacerla depender de una decisión arbitraria, como sucede en el caso de Parfit. Lo que sí implicaría es que la identidad personal dependería de una convención. Sin embargo, las convenciones no son necesariamente arbitrarias. Lo que determina que algo sea una convención es que sea el resultado de un acuerdo ante al menos dos alternativas. Si no hay motivos para preferir ninguna alternativa, la convención será arbitraria. Pero puede haber buenos motivos para preferir una alternativa sobre el resto (vid. Gilbert, 1989: 340-341). Y cuando éste es el caso, es posible justificar una decisión moral con base en dicha convención.

Creo que el caso que nos ocupa es de este tipo. Ante situaciones en las que la identidad personal es indeterminada, puede eliminarse dicha indeterminación eligiendo una entre las varias opciones disponibles. Lo que diferencia mi propuesta de la solución de Parfit es que, en mi caso, la negociación narrativa que eliminaría dicha indeterminación tendría como tarea precisamente la de encontrar buenas razones para preferir una alternativa sobre las otras. De este modo, la identidad dependería de una convención, pero no de una decisión arbitraria.

De acuerdo con Parfit, ante un caso como el de Bill no habría ninguna razón ni física ni psicológica que pudiese justificar una decisión sobre las identidades de BillC1 y BillC2 (Parfit, 1984: 241). Sin embargo, creo que Parfit se equivocaba, pues creo que podría haber razones para hacerlo. Por otro lado, la tesis social de la narratividad añade una dimensión social a la identidad personal que también 
podría ser fuente de razones para tomar dicha decisión. Dado que agotar todas las razones que podrían darse en la negociación narrativa correspondiente ocuparía más espacio del disponible, me limitaré a ofrecer algunos ejemplos.

Empezando por las razones físicas, debemos considerar que el cerebro (esto es, una parte del encéfalo superior) tiene dos hemisferios que en principio pueden dividirse. Sin embargo, las estructuras del encéfalo medio e inferior no son divisibles. Siendo así, el caso de Bill sólo sería posible si nos limitásemos a trasplantar el encéfalo superior de Bill, presuponiendo que los encéfalos medios e inferiores de sus hermanos estén en buenas condiciones. Pero si los encéfalos medios e inferiores de sus hermanos están en buenas condiciones, jsignifica que todavía están vivos! Para poder establecer un diagnóstico de muerte cerebral es necesario que la totalidad del encéfalo de una persona haya dejado de funcionar. Si no es así, entonces los hermanos de Bill son todavía personas vivas, cuyas familias podrían negarse -y con razón- a que los matasen para tratar de salvarle la vida a Bill. Otra posibilidad sería que uno de los hermanos de Bill tuviese todavía los encéfalos medio e inferior intacto, mientras que el otro estuviese en muerte cerebral. Si en este caso uno de los hermanos de Bill recibiese un hemisferio cerebral de Bill y el otro recibiese el otro hemisferio y las partes media e inferior del encéfalo de Bill, entonces habría una diferencia física importante entre BillC1 y BillC2, lo que podría llevarnos a concluir que, en todo caso, sería el cuerpo que ha recibido también los encéfalos medio e inferior de Bill el único que tendría derecho a reclamar la identidad de Bill.

Con respecto a las razones psicológicas que podríamos encontrar, Parfit parte del supuesto de que los dos hemisferios cerebrales de una persona podrían ser funcionalmente idénticos. Sin embargo, como señalábamos más arriba, se trata de un supuesto altamente cuestionable. Actualmente las neurociencias indican que distintas partes del cerebro son responsables de distintas funciones cognitivas. En este sentido, lo más probable es que las propiedades mentales de BillC1 y BillC2 sean distintas entre sí. Podría ser que uno de ellos se sintiese idéntico a Bill, mientras que el otro no. O puede ser que uno tenga la capacidad del lenguaje y el otro no. O que uno de ellos sea una persona medianamente funcional mientras que el otro sea incapaz de realizar ninguna de las actividades que normalmente las personas realizamos de manera independiente en nuestro día a día. Podemos ver así que incluso aunque pudiésemos trasplantar los dos hemisferios cerebrales a los dos hermanos de Bill, podríamos encontrar buenas razones psicológicas para establecer diferencias entre BillC1 y BillC2 y poder decidir así quién de ellos es idéntico a Bill. 
Finalmente, con respecto a las razones sociales, podemos pensar en la actitud que los familiares de Bill podrían tener hacia el caso. A lo mejor la familia de Bill es muy religiosa, y considera que la muerte cerebral de los dos hermanos de Bill no es suficiente para considerarles verdaderamente muertos. Si esto fuese así, podrían decidir que ni BillC1 ni BillC2 son idénticos a Bill, sino que mantienen las identidades de los hermanos de Bill. Si esta actitud estuviese muy extendida en el entorno inmediato de Bill, ni BillC1 ni BillC2 podrían desempeñarse como si fuesen Bill en ningún contexto, dándonos motivos para pensar que Bill, en realidad, no sobrevivió a la operación.

Los ejemplos que acabamos de revisar muestran que podrían existir muchas razones, de índole tanto física como psicológica o social, para tomar una decisión al respecto de las identidades de BillC1 y BillC2. Siendo así, la determinación de la identidad no se conseguiría a través de una decisión arbitraria, sino a través de un consenso fundamentado en buenas razones.

Alguien podría objetar que para justificar la importancia de la identidad personal no es suficiente con mostrar que ésta no depende de una decisión arbitraria. Si nuestra identidad nos importa lo hace desde un punto de vista subjetivo, y es desde ese punto de vista subjetivo, desde nuestra manera particular de experimentar nuestra existencia, desde donde se debe justificar dicha importancia.

Para dar respuesta a esta objeción es necesario recordar lo que dijimos al introducir las teorías narrativas de la identidad. Estamos hablando de una postura neo-lockeana para la cual lo que determina la identidad personal a lo largo del tiempo es la existencia continua de un mismo sujeto de experiencias que mantiene una conciencia unificada. En este sentido, la negociación narrativa que constituye nuestra identidad no parte de una posición de enfrentamiento entre lo que cada uno piensa de sí mismo y lo que dicen los demás. Dicha negociación tiene lugar, sobre todo, en nuestra manera de comprendernos a nosotros mismos. Las narraciones de los demás imponen restricciones a la manera en que podemos entendernos a nosotros mismos, de tal manera que influyen en nuestra propia narración de una manera tanto consciente como inconsciente. En este sentido, cuando afirmo que una ley sobre los casos de duplicaciones en identidad personal podría ayudarnos a solucionar la indeterminación que se puede producir en dichos casos sin comprometernos con la tesis de que la identidad no es lo que importa en nuestra supervivencia, lo que quiero decir es que dicha ley podría influir en nuestras creencias sobre las condiciones en las que podríamos sobrevivir en tanto sujetos de experiencias. De este modo, nuestra manera de enfrentarnos 
a dichos casos de duplicación sería experimentada directamente de acuerdo a lo establecido en la ley, y no como una imposición desde el exterior.

En este punto alguien podría objetar que una ley no podría cambiar sus opiniones al respecto de su continuidad a lo largo del tiempo. Efectivamente, algo así podría suceder. Sin embargo, hemos de tener en cuenta algunos aspectos. Primero, nos encontramos con un tema que posiblemente no ha sido objeto de reflexión por parte de nadie más allá de un reducido número de filósofos. Encontrar a alguien que tuviese opiniones firmes e inalterables sobre cómo afectaría a su supervivencia un caso de duplicación es algo altamente improbable y propio, en todo caso, de alguna minoría. Segundo, todas las convenciones son víctimas de este problema. De vez en cuando las noticias presentan a alguien que ha decidido conducir en sentido contrario en una autopista. Sin embargo, una convención no se ve afectada por el hecho de que una minoría decida no respetarla, siempre y cuando se trate efectivamente de una minoría y existan métodos adecuados para castigar la falta de respeto a la convención. En este sentido es pertinente señalar de nuevo lo extraño que será encontrar a alguien con opiniones tan firmes sobre el problema de la duplicación que se niegue a modificar sus creencias. Por último, no es mi trabajo señalar cuál sería la mejor forma de castigar a aquellos que no respeten una hipotética convención que resuelva el problema de la duplicación. Pero sí puedo señalar lo difícil que sería negarse a aceptar una convención de este tipo. Imaginemos que, por los motivos que sean, nuestra convención indica que BillC1 es idéntico a Bill. Negarse a aceptar esta convención implicaría negarse a reconocer a BillC1 la identidad de Bill y tratarle como si fuese otra persona. Pero empezar a tratar a las personas como si fuesen alguien diferente a quienes todos los demás dicen es un comportamiento peligroso que conlleva el riesgo de que consideren que hemos perdido la razón. Como un padre que se negase a aceptar que su hijo ha fallecido al tener muerte cerebral, alegando que los criterios para el diagnóstico de la muerte cerebral son convencionales, e insistiese en llevarse el cuerpo de su hijo a casa.

\section{Un posible problema desde la filosofía experimental}

En los últimos años la filosofía experimental ha venido cuestionando el papel que los filósofos dan a la intuición en sus investigaciones. Considerando que mi artículo concede una importancia capital a dos intuiciones sobre el problema de la identidad personal -que la identidad personal es determinada y que la 
identidad personal es lo que importa en nuestra supervivencia- resulta pertinente preguntarse si las reflexiones que han venido haciendo los filósofos experimentales sobre este tema afectan en alguna medida a mi trabajo.

En primer lugar, debemos distinguir entre dos tipos de críticas que se pueden encontrar en la filosofía experimental (vid. Knobe \& Nichols, 2017). Por un lado, están aquellos que señalan que las intuiciones no son fiables, pues varían de una persona a otra en función de factores tanto sociales como individuales. En este sentido, abogan por el abandono de las intuiciones como herramienta para la reflexión filosófica. Por otro lado, algunos han señalado que, aunque las intuiciones pueden ser de utilidad para la reflexión filosófica, los filósofos frecuentemente generalizan a partir de su propio caso, asumiendo que todo el mundo comparte sus intuiciones. Al considerar esto como un error, este segundo tipo de filósofos experimentales defiende que las propias intuiciones del filósofo deben ser sustituidas por las intuiciones que verdaderamente posee la gente, siendo necesario realizar estudios empíricos para descubrir cuáles son dichas intuiciones.

Con respecto a la primera crítica, pertenece a un debate que escapa del objeto de este artículo. Simplemente señalaré que ha habido excelentes respuestas al mismo principalmente basadas en dos puntos: (1) la variabilidad de las intuiciones de una persona a otra no es esencialmente problemática, dado que las intuiciones pueden tener un valor epistémico distinto según quién las posea; y (2) la variabilidad en las intuiciones puede achacarse en gran medida a desacuerdos verbales sobre el significado de los términos involucrados y el contexto en el que se emiten (vid. e.g. Shieber, 2010; Sosa, 2007). En cualquier caso, no entraré más a fondo en este debate, por lo que únicamente seńalo mi postura a favor del uso de las intuiciones en la discusión filosófica y remito al lector interesado a la bibliografía especializada.

Donde sí querría detenerme un poco más es en el segundo tipo de críticas que los filósofos experimentales han realizado al uso de las intuiciones. Aunque ha habido múltiples trabajos de filosofía experimental en relación al problema de la identidad personal, ninguno de ellos ha estado referido específicamente a las dos intuiciones que nos han ocupado en este artículo (vid. Shoemaker \& Tobia, forthcoming: 15-25). No obstante lo anterior, podemos preguntarnos si una encuesta destinada a detectar lo que la mayoría de la población intuye sobre la importancia de la identidad en la supervivencia y sobre la determinación de la identidad debería preocuparnos. Al fin y al cabo, el único motivo que esgrimo para tratar de defender ambas tesis es que son dos intuiciones muy fuertes. Pero 
¿no depende su fuerza de que efectivamente sean intuiciones extendidas entre la mayoría de la gente?

Para poder responder a esa pregunta debemos primero aclarar qué es lo que entiendo por la fuerza de una intuición. Con esa expresión me refiero a su valor epistémico. Cuando una intuición tiene un mayor valor epistémico que otra, tiene más fuerza. Bajo este supuesto, no creo que la fuerza de una intuición dependa necesaria o únicamente de la cantidad de gente que la comparte. En este sentido podemos recordar una de las respuestas que se han ofrecido a las críticas de la filosofía experimental: algunas intuiciones tienen un mayor valor epistémico que otras, dependiendo de quien las posea. Pongamos un ejemplo. Si tenemos un dolor en el vientre, todos podemos tener alguna intuición acerca de qué puede estar causando dicho dolor. Pero las intuiciones de un gastroenterólogo no estarían en un mismo nivel epistémico que las nuestras. Nos haríamos un flaco favor si, ante nuestro dolor, en lugar de fiarnos de la intuición del médico, exigiésemos que nos diesen quimioterapia porque todo el mundo en nuestra familia piensa que tenemos cáncer de estómago. Del mismo modo, todo el mundo puede tener alguna intuición sobre un problema filosófico. Pero los filósofos profesionales poseen conocimientos específicos sobre el tema, así como ańos de entrenamiento en la reflexión filosófica. Sus conocimientos y su forma de abordar los problemas parecen conceder un valor epistémico a sus intuiciones que, en principio, excede al valor que pueda tener una intuición contraria sostenida por otras personas.

A pesar de ello, no creo que la fuerza de las intuiciones que defiendo en este artículo sea totalmente independiente de lo que intuye la mayoría de la gente. Como he señalado a lo largo del texto, la tesis social de la narratividad añade una dimensión social al problema de la identidad personal. De esta manera, la identidad personal queda constituida en parte por las creencias sociales que existen acerca de las personas. En consecuencia, si una encuesta detectase que la mayoría de la gente rechaza alguna de las dos intuiciones en las que baso mi artículo -o ambas- sería un motivo para pensar que los filósofos están fallando al detectar cuáles son las creencias sociales que existen acerca de las personas. No tengo ninguna razón para pensar que éste podría ser el caso, pues en mi experiencia una inmensa mayoría de la gente suele estar de acuerdo con ambas intuiciones. El hecho de que no haya estudios al respecto desde la filosofía experimental también podría hacernos pensar que ningún filósofo ha pensado que las intuiciones de la gente podrían diferir de las de los filósofos en estos dos puntos. En cualquier caso, si efectivamente se demostrase que la gente no comparte estas intuiciones, habría que valorar en qué porcentaje y por qué motivos se da 
ese rechazo para calibrar su valor epistémico. Si su valor epistémico fuese mayor que el de las intuiciones de los filósofos, nos encontraríamos ante un escenario verdaderamente revolucionario para la investigación sobre el problema de la identidad personal. De todas maneras, como ya he señalado, no tengo ningún motivo para sospechar que ése podría ser el caso.

\section{Conclusiones}

A lo largo del artículo he argumentado a favor de dos tesis. Primero, defendí que las teorías narrativas deben comprometerse con la defensa de que la identidad personal es determinada. El motivo para ello es que su origen se encuentra en el intento de ofrecer una alternativa a la contraintuitiva propuesta de Parfit, por lo que los narrativistas necesitan que su propuesta tenga un mayor contenido intuitivo que la del autor de Reasons and Persons. En segundo lugar, expliqué cómo los narrativistas pueden alcanzar dicho objetivo mediante una correcta interpretación de uno de sus postulados básicos, la tesis social de la narratividad. Dicha tesis establece que la identidad personal se constituye a través de una negociación narrativa entre nuestra propia narración vital y las narraciones que los demás tienen sobre nosotros. Otorgando un peso adecuado a ambos tipos de narraciones es posible encontrar un equilibrio que nos permita establecer una convención que evite la indeterminación de la identidad personal en los casos de duplicación. Tras ello, considerando el vínculo entre la intuición de que la identidad personal es determinada y la intuición de que la identidad personal es lo que importa en la supervivencia, argumenté que para los narrativistas la defensa de la primera intuición es compatible con la segunda. Finalmente, mostré cómo la filosofía experimental podría desafiar las conclusiones de este artículo y por qué no hay motivos para pensar que dicho desafío pueda llegar a suceder.

\section{Agradecimientos}

Agradezco a Fernando Broncano y dos revisores anónimos sus comentarios sobre versiones previas de este artículo. 


\section{Bibliografía}

Broncano, Fernando (2013). Sujetos en la niebla: Narrativas sobre la identidad. Barcelona: Herder.

Bruner, Jerome S. (1986). Actual Minds, Possible Worlds. Cambridge, Mass.: Harvard University Press.

Cruz, Manuel (2005). Las malas pasadas del pasado: Identidad, responsabilidad, historia. Barcelona: Anagrama.

Dennett, Daniel C. (1991). Consciousness Explained. New York, Boston and London: Little, Brown and Company.

Gilbert, Margaret (1989). On Social Facts. Princeton, NJ: Princeton University Press.

Goldie, Peter (2012). The Mess Inside: Narrative, Emotion, and the Mind. Oxford: Oxford University Press.

Jongepier, Fleur (2016). "Towards a constitutive account of implicit narrativity". Phenomenology and the Cognitive Sciences, vol. 15/1, pp. 51-66.

Knobe, Joshua, \& Nichols, Shaun (2017). Experimental Philosophy. En E. N. Zalta (Ed.), The Stanford Encyclopedia of Philosophy (Winter 2017 Edition). Recuperado de https://plato.stanford.edu/archives/win2017/entries/experimental-philosophy/.

Lamarque, Peter (2007). On the Distance between Literary Narratives and Real-Life Narratives. En D. D. Hutto (Ed.), Narrative and Understanding Persons. Cambridge: Cambridge University Press, pp. 117-132.

Lindemann, Hilde (2014). Holding and Letting Go: The Social Practice of Personal Identities. Oxford: Oxford University Press.

Locke, John (1975). An Essay Concerning Human Understanding. Oxford: Oxford University Press.

Macintyre, Alasdair (2007). After Virtue: A Study in Moral Theory. Notre Dame, IN: University of Notre Dame Press.

Parfit, Derek (1984). Reasons and Persons. Oxford: Oxford University Press.

Ricoeur, Paul (1994). Oneself as Another. Chicago: University of Chicago Press.

Rudd, Anthony (2012). Self, Value, and Narrative: A Kierkegaardian Approach. Oxford: Oxford University Press.

Sanfélix, Vicente (1994). Las personas y su identidad. Logos: Anales del seminario de metafísica, vol. 28, pp. 257-285.

Schechtman, Marya (1996). The Constitution of Selves. Ithaca, NY: Cornell University Press. 
- (2014). Staying Alive: Personal Identity, Practical Concerns, and the Unity of a Life. Oxford: Oxford University Press.

Shieber, Joseph (2010). On the nature of thought experiments and a core motivation of experimental philosophy. Philosophical Psychology, vol. 23/4, pp. 547-564.

Shoemaker, David, \& Tobia, Kevin (forthcoming). Personal Identity. En M. Vargas \& J. M. Doris (Eds.), The Oxford Handbook of Moral Psychology. Oxford: Oxford University Press.

Sosa, Ernest (2007). Experimental philosophy and philosophical intuition. Philosophical Studies, vol. 132/1, pp. 99-107.

Strawson, Galen (2008). Against Narrativity. En Real Materialism and Other Essays. Oxford: Oxford University Press, pp. 189-207.

- (2012). We Live Beyond Any Tale That We Happen to Enact. Harvard Review of Philosophy, vol. 18, pp. 73-90.

Taylor, Charles (2001). Sources of the Self: The Making of Modern Identity. Cambridge, Mass.: Harvard University Press.

Wilkes, Kathleen V. (1988). Real People: Personal Identity without Thought Experiments. Oxford: Clarendon Press.

Williams, Bernard (1970). The Self and the Future. The Philosophical Review, vol. 79/2, pp. 161-180.

Recibido: $29 / 11 / 2018$

Aceptado: 22/04/2019

Este trabajo se encuentra bajo una licencia de Creative Commons ReconocimientoNoComercial-SinObraDerivada 4.0 
